\title{
Portal Dissemination of Fusarium graminearum in a Patient with Acute Lymphoblastic Leukemia and Febrile Neutropenia
}

\author{
Mary Gabriela Uscamayta ${ }^{1}$, Alexandra Martin-Onraet ${ }^{1}$, Karla Espinosa-Bautista ${ }^{2}$, Roberto Herrera-Goepfert ${ }^{3}(\mathbb{D}$, \\ Rigoberto Hernández-Castro ${ }^{4}$ and Carolina Perez-Jimenez ${ }^{1, *(1)}$ \\ 1 Infectious Disease Department, National Institute of Cancer, Mexico City 14080, Mexico; \\ gabriela-mary@hotmail.com (M.G.U.); alexitemaon@gmail.com (A.M.-O.) \\ 2 Hematology Department, National Institute of Cancer, Mexico City 14080, Mexico; karlaadrianae@gmail.com \\ 3 Pathology Department, National Institute of Cancer, Mexico City 14080, Mexico; rhgoepfert@gmail.com \\ 4 Ecology of Pathogen Agents Department, General Hospital Manuel Gea González, \\ Mexico City 14080, Mexico; rigo37@gmail.com \\ * Correspondence: capeji@hotmail.com
}

check for

updates

Citation: Uscamayta, M.G.;

Martin-Onraet, A.; Espinosa-Bautista,

K.; Herrera-Goepfert, R.;

Hernández-Castro, R.; Perez-Jimenez,

C. Portal Dissemination of Fusarium

graminearum in a Patient with Acute

Lymphoblastic Leukemia and Febrile

Neutropenia. Infect. Dis. Rep. 2021, 13 ,

11-17. https://doi.org/10.3390/

idr13010002

Received: 29 October 2020

Accepted: 2 December 2020

Published: 1 January 2021

Publisher's Note: MDPI stays neutral with regard to jurisdictional clai$\mathrm{ms}$ in published maps and institutional affiliations.

Copyright: (C) 2021 by the authors. Licensee MDPI, Basel, Switzerland. This article is an open access article distributed under the terms and conditions of the Creative Commons Attribution (CC BY) license (https:// creativecommons.org/licenses/by/ $4.0 /)$.

\begin{abstract}
We present the case of a man with acute lymphoblastic leukemia and prolonged profound neutropenia, who developed an invasive infection by Fusarium graminearum, acquired via noncutaneous entry, with gastrointestinal symptoms, sigmoid perforation and liver abscesses due to portal dissemination. The etiologic agent was identified using the 18S-ITS1-5.8S-ITS2-28S rRNA sequence gene, from a liver biopsy. The infection was resolved with surgical drainage and antifungal treatment based on voriconazole. As far as we know, there are no previous reports in the literature of cases of human infection due to Fusarium graminearum.
\end{abstract}

Keywords: Fusarium graminearum; portal dissemination; invasive fungal infection; rRNA sequence gene; febrile neutropenia

\section{Introduction}

In the last decades, there has been an increase in the incidence of invasive fungal infections (IFI), related to new immunosuppressive treatments and improved diagnosis with new molecular techniques and higher diagnostic yields [1,2]. We present the case of a male patient with acute lymphoblastic leukemia and prolonged profound neutropenia, who developed an invasive infection by Fusarium graminearum, acquired via non-cutaneous entry, presenting an atypical clinical course, gastrointestinal symptoms, sigmoid perforation and liver abscesses due to portal dissemination. The etiologic agent was identified using the 18S-ITS1-5.8S-ITS2-28S rRNA sequence gene from a liver biopsy. This is the first reported case of portal dissemination by Fusarium genus and the first report of Fusarium graminearum infection in humans.

\section{Ethical Considerations}

The case we submit for publication is about a single patient who provided his verbal consent for this purpose. We do not include any details or information that could lead to patient identification

\section{Case Report}

Male patient, 26 years old, kitchen assistant, originated from Taxco, Guerrero and living there until he emigrated to Texas, United States, in 2016, where he was diagnosed with B lymphoblastic leukemia in January 2017. He received four cycles of unspecified chemotherapy with apparent remission of the disease and came back to Mexico (Cuernavaca, Morelos). He was admitted to the National Institute of Cancer in Mexico City in 
November 2017, where he was diagnosed with early relapse. From November 2017 to January 2018, he received chemotherapy for relapse with BFM like protocol (L-Asparaginase, Vincristine, Daunorubicin, and Prednisone). He did not receive any antifungal prophylaxis. On day +23 he was presented with febrile neutropenia, with no etiologic agent identified. An empirical treatment of five days of Meropenem was administered, with resulting in fever remission. He was discharged 6 days after his admission, asymptomatic but with persistent neutropenia.

Two days later, he had come back with an acute abdomen, although afebrile. Vital constants at admission were: $\mathrm{BP}: 107 / 78 \mathrm{mmHg}$, $\mathrm{HR}: 90 \mathrm{bpm}, \mathrm{RR}: 20 \mathrm{rpm}, \mathrm{T}^{\circ}: 36.5^{\circ} \mathrm{C}$, weight: $55 \mathrm{Kg}$, height: $1.62 \mathrm{~m}$, BMI: $21 \mathrm{Kg} / \mathrm{m} 2$. His blood tests showed leukocytes of $0.32 \times 10^{3} / \mu \mathrm{L}$, absolute neutrophils $0.0032 \times 10^{3} / \mu \mathrm{L}$, hemoglobin $5.7 \mathrm{~g} / \mathrm{dL}$, platelets $35 \times 10^{3} / \mu \mathrm{L}$, creatinine $0.66 \mathrm{mg} / \mathrm{dL}$, ALT $174 \mathrm{IU} / \mathrm{L}$, AST $82 \mathrm{IU} / \mathrm{L}$, ALP $1004 \mathrm{IU} / \mathrm{L}$, GGT $572 \mathrm{IU} / \mathrm{L}$, total bilirubin $8 \mathrm{mg} / \mathrm{dL}$, conjugated bilirubin $6.4 \mathrm{mg} / \mathrm{dL}$, unconjugated bilirubin $1.62 \mathrm{mg} / \mathrm{dL}, \mathrm{LDH}$ $287 \mathrm{IU} / \mathrm{L}$, albumin $1.7 \mathrm{mg} / \mathrm{dL}, \mathrm{C}$ reactive protein $8.4 \mathrm{mg} / \mathrm{dL}$. The patient underwent exploratory laparotomy which revealed sigmoid perforation. Sigmoidectomy and colostomy were performed. The initial pathology report was diverticulum perforation. Forty-eight hours later, he presented moderate diffuse abdominal pain, and temperature $>38^{\circ} \mathrm{C}$. The blood cultures were negative. Administration of Meropenem, caspofungin and Filgastrim was empirically initiated. Contrast abdominal tomography (CT) showed diffuse liver lesions consistent with liver abscesses (Figure 1).

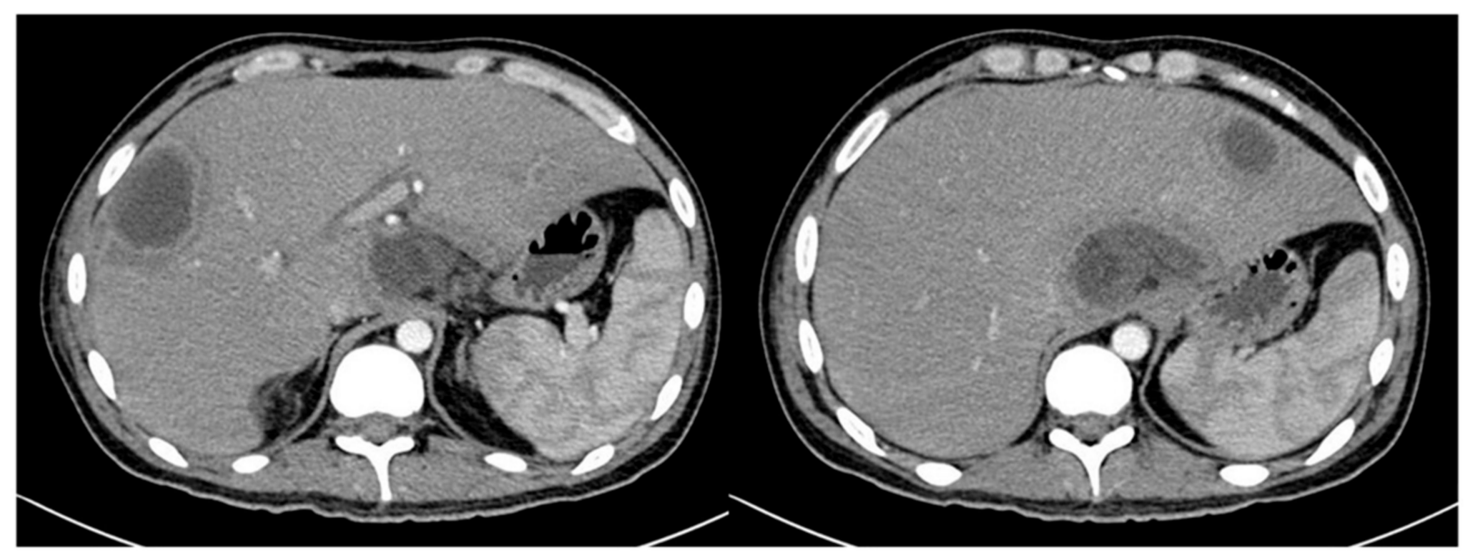

Figure 1. Abdominal CT scan with intravenous contrast, which shows: Diffuse and poorly defined hypodense lesions, one located in the liver segment II measuring $3.4 \mathrm{~cm}$, another located in the caudate lobe measuring $7 \mathrm{~cm}$, and another located in the hepatic segment VII with extension to segment VI measuring $7 \mathrm{~cm}$; consistent with liver abscesses.

A liver biopsy of the lesions was performed under ultrasound guidance; the aerobic, anaerobic and Sabouraud dextrose agar cultures were negative. The pathology showed granulomatous hepatitis with extensive necrosis; Grocott's stain evidenced hyaline hyphae. The histopathology of the sigmoid surgical specimen was reviewed again and then reported as transmural ischemic necrosis, acute and chronic peritonitis, and positive Grocott's stain with the same hyphae described in the liver (Figure 2).

Hyalohyphomycosis was concluded. The serum galactomannan was negative. Pulmonary involvement was not seen by tomography. The antifungal treatment was changed from caspofungin to intravenous Voriconazole on day 23. The patient had gradual clinical improvement the neutropenia was remitted and was discharged with a treatment of oral voriconazole $200 \mathrm{mg}$ bid. He was, at that time, in complete remission of leukemia. 


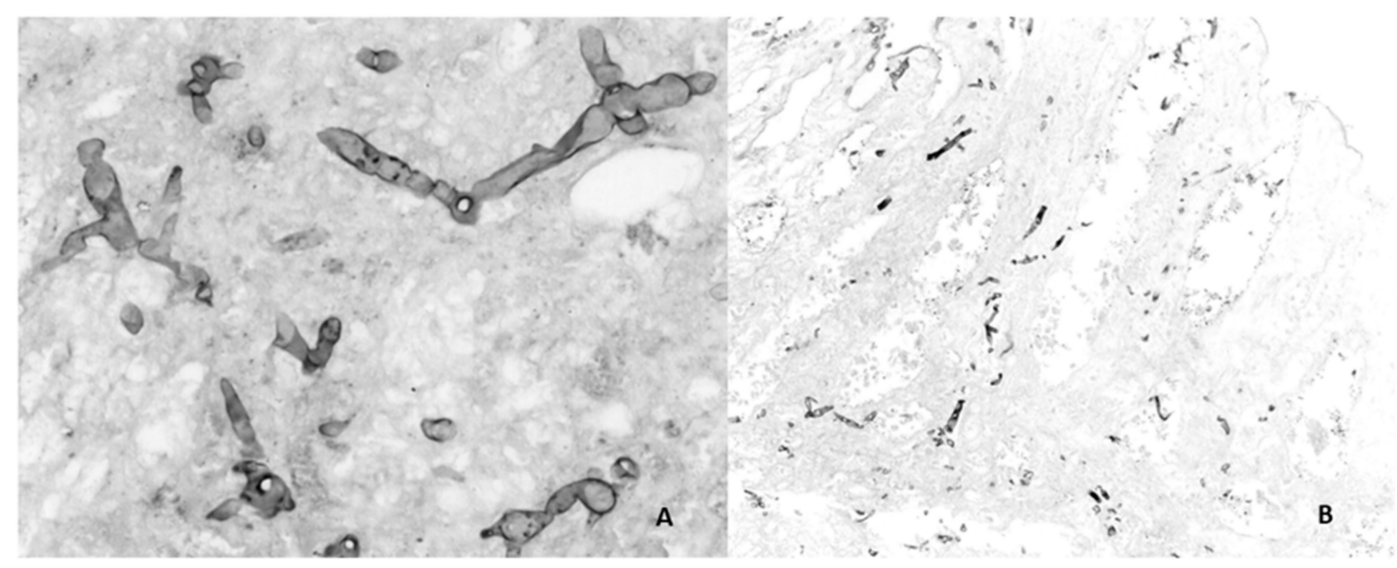

Figure 2. (A) Non-pigmented, separated hyphae with acute angle branching in liver biopsy tissue, Grocott methenamine silver-stained specimen, $40 \times$. (B) Non-pigmented, separated hyphae with acute angle branching in intestinal tissue, Grocott methenamine silver-stained specimen, $10 \times$.

Three weeks later he was readmitted for abdominal pain in the right hypochondrium with fever without neutropenia; he reported bad adherence to oral antifungal treatment for economic reasons. The new abdominal CT showed the persistence of liver abscesses. Exploratory laparotomy plus drainage were performed finding abscesses in segments II, IV and VI. The anaerobic culture grew Bacteroides fragilis; he completed 14 days of Metronidazole. The fungal culture was negative. The pathology report was extensive granulomatous hepatitis with necrosis and negative Grocott's stain. The patient received 2 weeks of intravenous Voriconazole plus 6 weeks of the same oral antifungal. At the end of treatment, the abdominal CT showed only one residual lesion of $16 \mathrm{~mm}$. A timeline of the case is shown in Figure S1.

He did not present further complications of the infectious process. However, six months later, while undergoing hematopoietic stem-cell transplant evaluations, he presented a relapse of leukemia and he decided to stop the antineoplastic treatment. He died seven months later.

\section{Materials and Methods for Identification of the Causative Agent}

A paraffin block of the first liver biopsy was requested, and DNA extraction was performed. Genomic DNA was extracted from the paraffin-embedded tissue sample using a DNeasy blood and tissue kit (Qiagen, Ventura, CA, USA) according to the manufacturer's instructions before preliminary removal of paraffin by extraction with xylene protocol. The molecular identification was performed by 18S-ITS1-5.8S-ITS2-25S rRNA gene amplification using a set of primers previously reported to identify fungi species $\left(5^{\prime}\right.$ TCCGTAGGTGAACCTTGCGG-3') and ITS4 (5'-TCCTCCGCTTATTGATATGC-3'). A PCR product of $500 \mathrm{bp}$ was amplified, purified and sequenced in both directions; nucleotide sequence was determined with Taq FS Dye Terminator Cycle Sequencing FluorescenceBased Sequencing and analyzed on an Applied Biosystems 3730 DNA sequencing system (Foster City, CA, USA). The sequence was edited with Vector NTI program and homology search was performed in the GenBank database (nucleotide blast), finding a 100\% identity with Fusarium graminearum complex strain HUT59 [3]. (Figure 3). The sequences used are shown in Supplementary Figure S2. 
$1 \quad 2 \quad 3$

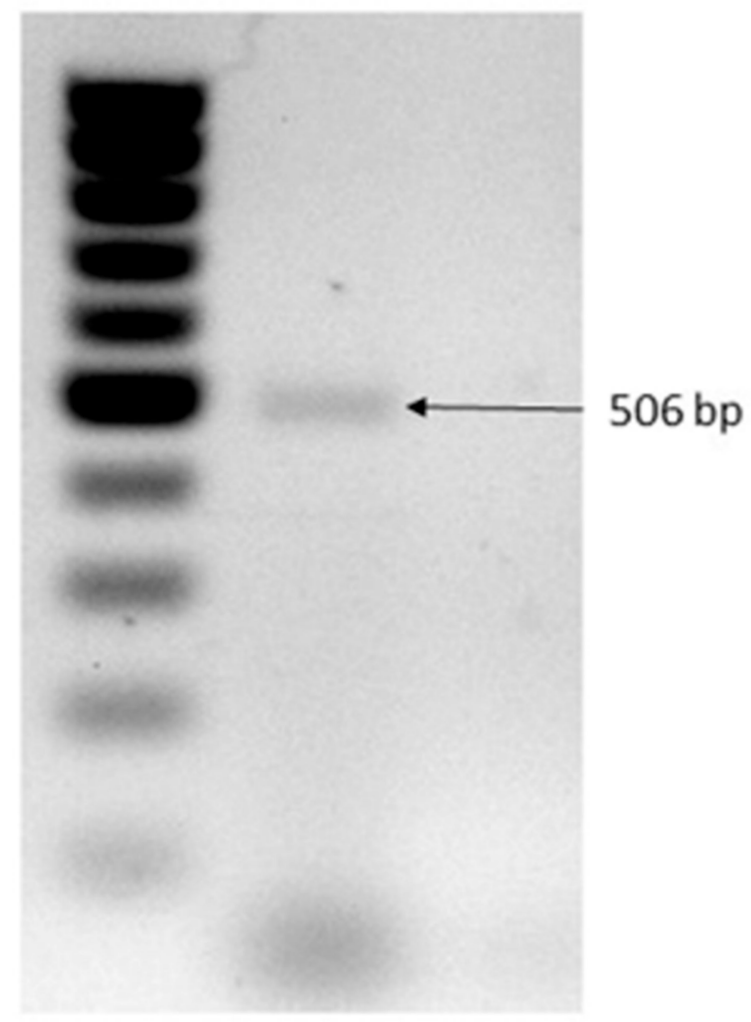

Figure 3. The 18S-ITS1-5.8S-ITS2-25S rRNA gene amplification. Lane 1: Molecular weight marker 100 bp DNA ladder; Lane 2: Liver biopsy sample; Lane 3: Negative control.

\section{Discussion}

In the last ten years, there has been an increasing incidence of disseminated filamentous fungal infections in patients with severe immunosuppression [1,2,4]. Hematological malignancies have a risk of $6.5 \%$ for IFI [5], mainly in the induction and consolidation phases. This increase is due to severe immunosuppression conditions by myelotoxic treatments and related clinical conditions but also to new technologies such as molecular biology $[6,7]$ that have increased the diagnosis ability.

Hialohifomycetes are opportunistic filamentous fungi that usually cause infection after environmental exposure [6]. Infection occurs through inhalation or skin breakdown (wounds, burns) [8]. Fusarium spp. is the second cause of infections by filamentous fungi after Aspergillus spp. More than 50 Fusarium species have been identified, but only about 12 are associated with infection in humans. F. incarnatum, F. moniliforme, F. oxysporum and $F$. solani usually cause allergic reactions and superficial infections such as keratitis and onychomycosis in immunocompetent hosts. In immunocompromised patients, it can cause from locally invasive diseases such as sinusitis and pneumonia, to fungemia and disseminated disease $[9,10]$. In neutropenic patients, it usually manifests as fever not responding to empirical antibiotics. Profound and prolonged neutropenia is the most important risk factor for dissemination and death due to fusariosis $[8,11]$. The most frequently isolated species in this setting are F. solani $(50 \%)$ and F. oxysporum $(20 \%)[6,8]$.

Fusarium graminearum plays a major role as the etiological agent of one important disease of small grain cereals and corn. First identified in 1884 in England, it is considered a major threat to wheat and barley [12]. The fungi, transmitted by seed, can survive up to 10 years as spores or resting structures in and on the seed. In Mexico, F. graminearum has been found in Hidalgo, Jalisco, Mexico, Michoacán and Tlaxcala [13-16]. This fungus can produce several mycotoxins, which have shown abortive estrogenic, gastric (nausea and vomiting) and neurotoxic toxicity in swine, cattle, dogs, cats, and ducks [16,17]. Grain that 
has been infected with the fungus can be incorporated into basic diets and could be a source of infection in humans. So far, no infections due to F. graminareum have been reported. Considering the patient's occupation (kitchen helper) and the high corn content in the Mexican diet, we supposed that the patient was colonized with F. graminearum through the daily ingestion and handling of corn. He possibly got the mould through oral ingestion, and symptoms were gastrointestinal due to portal dissemination, which explains why we did not document any fungemia or cutaneous infection, despite an invasive disease. Usually, in disseminated fusariosis, blood cultures are positive in more than $75 \%$ of cases $[6,8]$.

In our patient, the diagnosis of hyalohyphomycosis was first made by pathologic specimen. The biopsy revealed the presence of septate hyaline hyphae 3-6 $\mu \mathrm{m}$ diameter, indistinguishable from other hyalohyphomycosis such as Aspergillus, Acremonium [Cephalosporium], and Pseudallescheria [17]. Recently, the Mycotic Diseases Branch, from the Center for Disease Control and Prevention in Atlanta, described a new entity of invasive fungal infections (IFI), which they called "foodborne IFI", in a recently published systematic review [18], foodborne IFI are infections acquired through ingestion of food or water contaminated with the fungi causing the infection. Reports describe molds and yeasts as etiologic agents. Rhizopus sp is the most frequently described mold, but one case of IFI due to Fusarium moniliforme is reported in a man with acute lymphoblastic leukemia who ingested cereals. The diagnosis of foodborne IFI is difficult, clinical presentation is usually atypical, and mainly gastrointestinal. Fungi can spoil food that is ingested, and it is suggested that this category of fungal infections could be related to food insecurity. The source of the fungi should be identified to be able to classify the IFI as foodborne, which is often difficult to the ubiquitous nature of these fungi. As previously mentioned, although we suspect that our patient acquired the infection orally and this could correspond to foodborne IFI, we cannot corroborate the above because we could not have samples of the food that the patient ingested prior to his hospitalization and we did not perform stool cultures.

Today, comparative identification strategies based on gene sequencing can be considered the new gold standard for the identification of fungal species. Gene sequencing is characterized by PCR amplification of the ITS region, and sequencing of the resulting amplicon (s). The ITS region can be amplified reliably for most fungi [19]. These molecular biology techniques provide better discrimination of species morphologically indistinguishable from Fusarium spp, and can be performed on different types of materials, including paraffin blocks, which is why it is considered an emerging and promising methodology to be used in the routine identification of Fusarium spp. In our case, no other fungal species were identified in the studied specimen or in any other specimen from the patient. The Fusarium graminearum complex includes 13 species; biogeographic data suggest that the majority of the species within the complex have evolutionary origins in the Southern hemisphere and Asia. According to the surveys to date, F. austroamericanum, F. meridionale, F. cortaderiae, and F. brasilicum appear to be endemic to South America; F. acaciae-mearnsii to Australia or less likely Africa; F. asiaticum, F. vorosii and F. ussurianum to Asia; F. aethiopicum to Africa; F. boothii and F. mesoamericanum to Central America; F. gerlachii to the US and F. graminearum sensu stricto (F. graminearum s.s.) it is the most cosmopolitan species and has been found in Asia, Africa, America, Europe, and Oceania [20].

Finally, the treatment in localized disease can benefit from surgical debridement especially in disseminated disease together with the use of systemic antifungals. Fusarium species are relatively resistant in vitro to most antifungal agents, showing different susceptibility patterns between species. In general, most Fusarium isolates have lower inhibitory concentrations (MIC) for Amphotericin B higher than Aspergillus species. F. solani and F. verticillioides are usually resistant to azoles and they have higher MIC for azoles than other Fusarium species. In contrast, F. oxysporum and F. moniliforme may be susceptible to voriconazole and posaconazole [9]. Echinocandins show the highest MIC values [21]. As far as we know there are no previous reports in the medical literature of identification of F.graminearum as a pathogen in humans; therefore antifungal therapy was initiated based 
on is the antifungals reported in the literature for the Fusarium species and on the clinical response.

\section{Conclusions}

Prolonged profound neutropenia and alteration of the intestinal flora, secondary to myelotoxic chemotherapies and environmental exposure in patients with hematological neoplasms, are important risk factors for the development of invasive infections by filamentous fungi. This is the first reported case of invasive fungal infection by F. graminearum and the first case of portal dissemination of Fusarium spp. The anamnesis and exposure to environmental factors are important during clinical evaluation. F.graminearum was not identifiable by traditional methods such as culture and pathology. Access to molecular biology is extremely valuable to increase the performance of diagnostic tests in the identification of new species such as the F. graminearum reported in our paper.

Supplementary Materials: The following are available online at https:/ / www.mdpi.com/2036-744 $9 / 13 / 1 / 2 / s 1$, Supplementary material 1 (Figure S1) is a timeline of the present case. Supplementary material 2 (Figure S2) is a figure with the genetic sequences used for the identification of the etiologic agent (Fusarium graminearum complex).

Author Contributions: M.G.U. was in charge of the data curation of the patient; A.M.-O. and M.G.U. were in charge of the writing of the original manuscript; R.H.-C. was in charge of the methodology for the identification by molecular biology of the etiologic agent ; C.P.-J. was responsible for reviewing and editing the manuscript as well as submitting it for publication. R.H.-G. was in charge of the methodology for the identification of the agent in the pathology specimen. K.E.-B. also participated in the data curation of the patient and in the writing of the original manuscript.All authors have read and agreed to the published version of the manuscript.

Funding: No source of funding was used for the preparation of this report.

Institutional Review Board Statement: Not applicable. Due to the fact that our study is only descriptive (without any type of intervention) on the case of a single patient, and he provided his consent, the evaluation by the Institution's ethics committee was not considered necessary.

Informed Consent Statement: Informed consent was obtained from all subjects involved in the study.

Data Availability Statement: Data is contained within the article or supplementary material.

Acknowledgments: To all the infectious disease fellows who participated in the approach of this case, especially to Aaron Molina. And Rodrigo Toral-Villanueva for his technical support with the edition of figures.

Conflicts of Interest: All authors report having no conflict of interest.

\section{References}

1. Liu, Y.S.; Wang, N.C.; Ye, R.H.; Kao, W.Y. Disseminated Fusarium infection in a patient with acute lymphoblastic leukemia: A case report and review of the literature. Oncol Lett. 2014, 7, 334-336. [CrossRef] [PubMed]

2. Slavin, M.; Van Hal, S.; Sorrell, T.C.; Lee, A.; Marriott, D.J.; Daveson, K.; Kennedy, K.; Hajkowicz, K.; Halliday, C.; Athan, E.; et al. Invasive infections due to filamentous fungi other than Aspergillus: Epidemiology and determinants of mortality. Clin Microbiol Infect. 2015, 21, 490.e1-490.e10. [CrossRef] [PubMed]

3. Korabecna, M.; Liska, V.; Fajfrlík, K. PrimersITS1, ITS2 andITS4 detect the intraspecies variability in the internal transcribed spacers and 5.8S rRNA gene region in clinical isolates of fungi. Folia Microbiol. 2003, 48, 233-238. [CrossRef] [PubMed]

4. Wanke, B.; Lazéra, M.D.S.; Nucci, M. Fungal infections in the immunocompromised host. Memórias Inst. Oswaldo Cruz 2000, 95, 153-158. [CrossRef] [PubMed]

5. Pagano, L.; Girmenia, C.; Mele, L.; Ricci, P.; Tosti, M.E.; Nosari, A.; Buelli, M.; Picardi, M.; Allione, B.; Corvatta, L.; et al. Infections caused by filamentous fungi in patients with hematologic malignancies. A report of 391 cases by GIMEMA Infection Program. Haematologica 2001, 86, 862-870. [PubMed]

6. Fariñas, M.C.; Fernandez-Sampedro, M.; Armiñanzas, C. Clinical forms and treatment of infections caused by other filamentous fungi. Enferm. Infecc. Microbiol. Clin. 2012, 30, 414-419. [CrossRef] [PubMed]

7. Ruiz-Camps, I.; Jarque, I. Invasive mould disease in haematological patients. Rev. Iberoam. Micol. 2014, 31, 249-254. [CrossRef] [PubMed] 
8. Muhammed, M.; Anagnostou, T.; Desalermos, A.; Kourkoumpetis, T.K.; Carneiro, H.A.; Glavis-Bloom, J.; Coleman, J.J.; Mylonakis, E. Fusarium infection. Medicine 2013, 92, 305-316. [CrossRef] [PubMed]

9. Nucci, M.; Anaissie, E. Fusarium infections in immunocompromised patients. Clin. Microbiol Rev. 2007, 20, 695-704. [CrossRef] [PubMed]

10. Campo, M.; Lewis, R.E.; Kontoyiannis, D.P. Invasive fusariosis in patients with hematologic malignancies at a cancer center: 1998-2009. J. Infect. 2010, 60, 331-337. [CrossRef] [PubMed]

11. Nucci, M.; Anaissie, E.J.; Queiroz-Telles, F.; Martins, C.A.; Trabasso, P.; Solza, C.; Mangini, C.; Simoes, B.P.; Colombo, A.L.; Vaz, J.; et al. Outcome predictors of 84 patients with hematologic malignancies and Fusarium infection. Cancer 2003, 98, 315-319. [CrossRef] [PubMed]

12. Goswami, R.S.; Kistler, H.C. Heading for disaster: Fusarium graminearum on cereal crops. Mol. Plant Pathol. 2004, 5, 515-525. [CrossRef] [PubMed]

13. Ireta, M.J.; Gilchrist, L. Fusarium Head Scab of Wheat (Fusarium Graminareum Schwabe); CIMMYT: Texcoco, Mexico, $1994 ;$ p. 25.

14. Leyva-Mir, S.G.; Vega-Portillo, H.E.; Villaseñor-Mir, H.E.; Tlapal-Bolaños, B.; Vargas-Hernandez, M.; Camacho-Tapia, M.; TovarPedraza, J.M. Characterization of Fusarium species causing root rot of wheat in the Bajío, Mexico. Chil. J. Agric Anim Sci. 2017, 33, 142-151.

15. Sandoval-Martínez, E.; Leyva Mir, S.G.; Villaseñor-Mir, H.E.; Rodríguez-García, M.F.; Mariscal-Amaro, L.A. Diversity of fungi in grain of wheat of temporary. Rev. Mex. Fitopatol. 2012, 30, 145-149.

16. García-Aguirre, G.; Martinez-Flores, R. Fusarium species from corn kernels recently harvested and shelled in the fields in the Ciudad Serdán Region, Puebla. Rev. Mex. Biodivers. 2010, 81, 15-20.

17. Bonifaz, A. Hialohifomicosis y Otras Micosis Poco Frecuentes; McGraw-Hill: New York, NY, USA, 2010.

18. Benedict, K.; Chiller, T.M.; Mody, R.K. Invasive fungal infections acquired from contaminated food or nutritional supplements: A review of the literature. Foodborne Pathog. Dis. 2016, 13, 343-349. [CrossRef] [PubMed]

19. Jain, P.K.; Gupta, V.K.; Misra, A.K.; Gaur, R.; Bajpai, V.; Isaar, S. Current status of fusarium infection in human and animal. Asian J. Anim. Vet. Adv. 2011, 6, 201-227. [CrossRef]

20. Wang, J.H.; Ndoye, M.; Zhang, J.B.; Li, H.P.; Liao, Y.C. Population structure and genetic diversity of the Fusarium graminearum species complex. Toxins 2011, 3, 1020-1037. [CrossRef] [PubMed]

21. Taj-Aldeen, S.J. Reduced multidrug susceptibility profile is a common feature of opportunistic Fusarium Species: Fusarium multi-drug resistant pattern. J. Fungi 2017, 3, 18. [CrossRef] [PubMed] 